\title{
A Robust Photothermal Coating Strategy for Efficient Ice Removal
}

Yubo Liu ${ }^{a, b}$, Yang $W u^{* b, c}$, Yizhe Liu ${ }^{b}$, Rongnian $X u^{b}$, Shujuan Liu*a, Feng Zhou ${ }^{b}$

a State Key Laboratory of Solidification Processing, Centre of Advanced Lubrication and Seal Materials, Northwest Polytechnical University, Xi'an, Shaanxi, 710072, PR China.

${ }^{\mathrm{b}}$ State Key Laboratory of Solid Lubrication, Lanzhou Institute of Chemical Physics, Chinese Academy of Science, Gansu Lanzhou 730000, PR China.

C Qingdao Centre of Resource Chemistry and New materials, Shandong, Qingdao 266100, PR China.

*Corresponding Author. E-mail: yangwu@licp.cas.cn; liusj@nwpu.edu.cn 


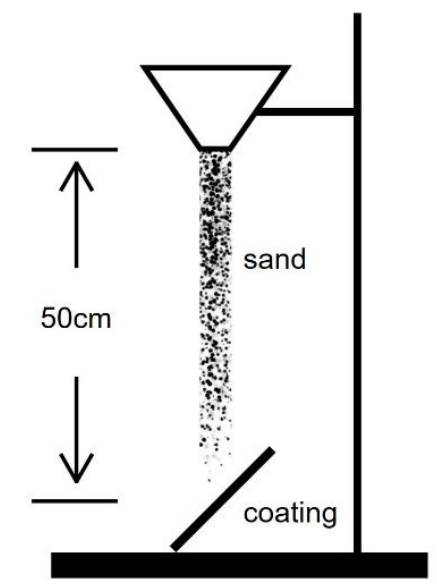

Figure S1 Schematic of sand corrosion test.

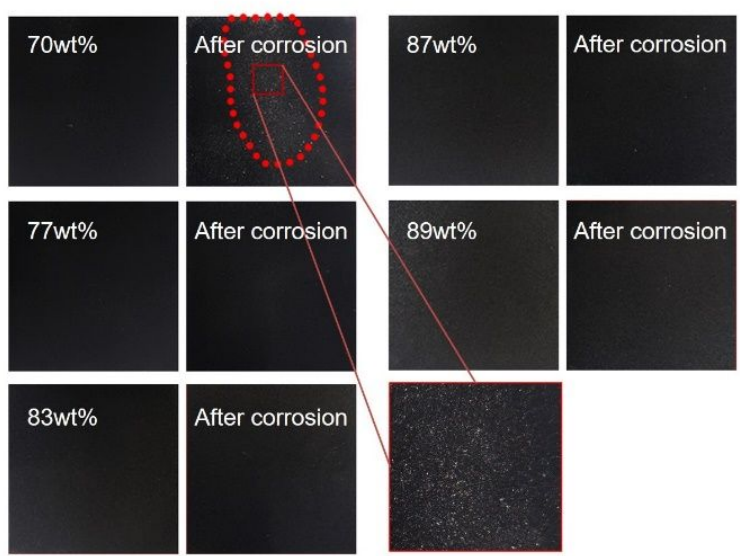

Figure S2 Photo images of coating surfaces after sand abrasion test.
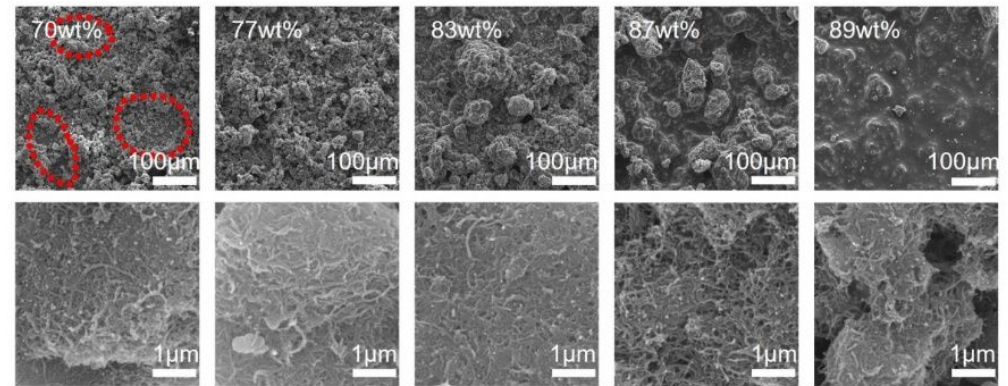

Figure S3 SEM images of coating surfaces after sand abrasion. 


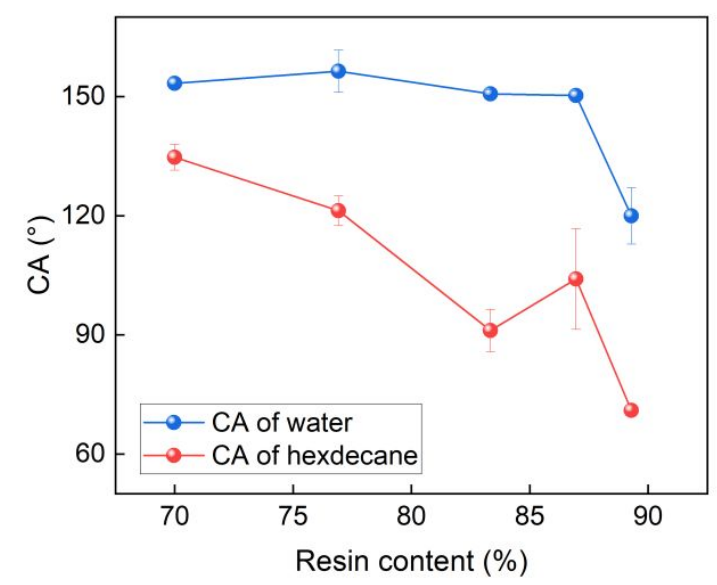

Figure S4 Contact angles of water and hexadecane on amphiphobic coating surface after sand abrasion.

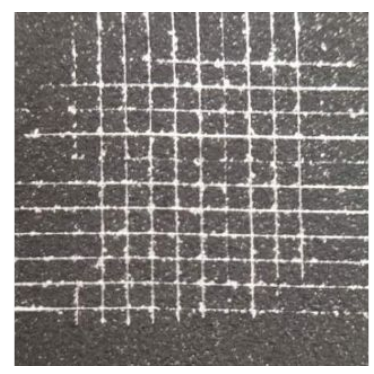

Figure S5 Photographs of coating surfaces after blades slashing.
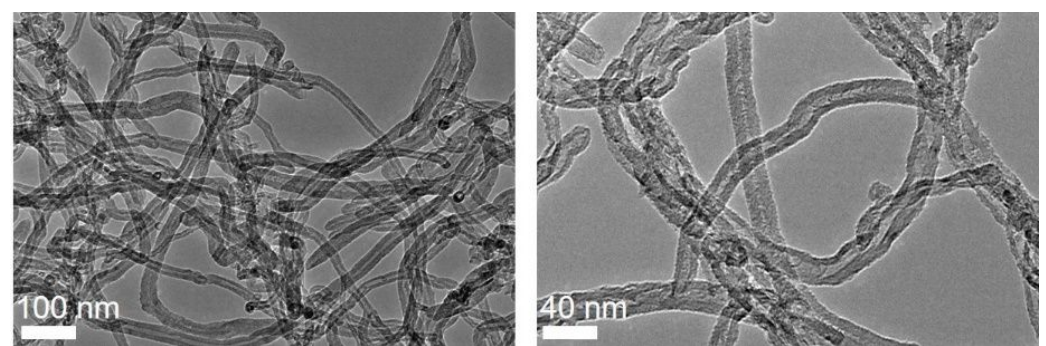

Figure S6 TEM images of raw MWCNTs at various amplification.
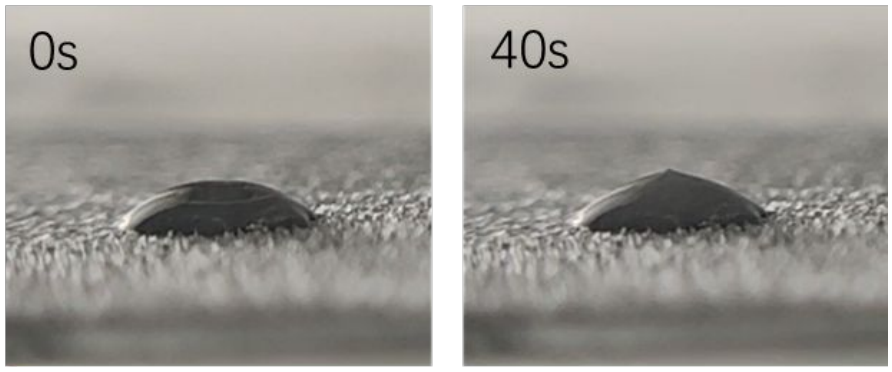

Figure S7 Water droplet freeze on hydrophilic photothermal coating after plasma etching within 40 s, which means the disappearance of anti-icing performance. 


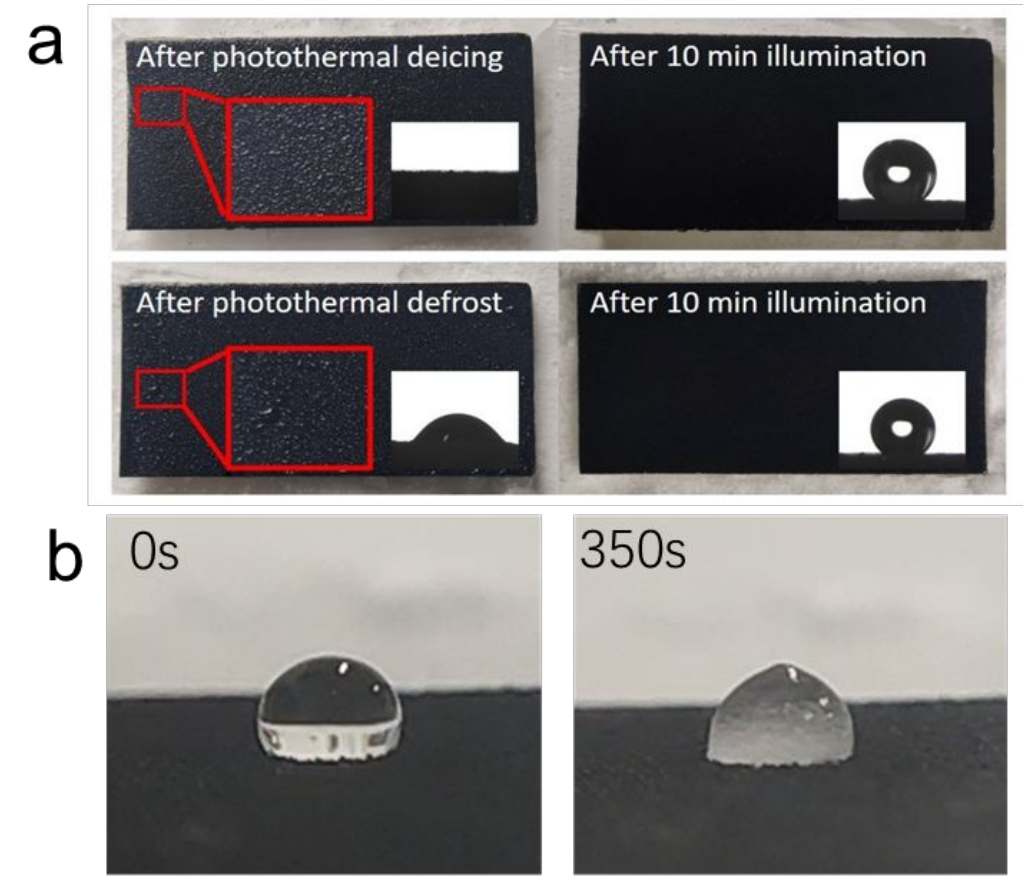

Figure S8 (a) Photo images and contact angles on surfaces after photothermal deicing/defrosting and after irradiation healing. (b) Droplet freeze on the coating surface after sunlight irradiation healing.

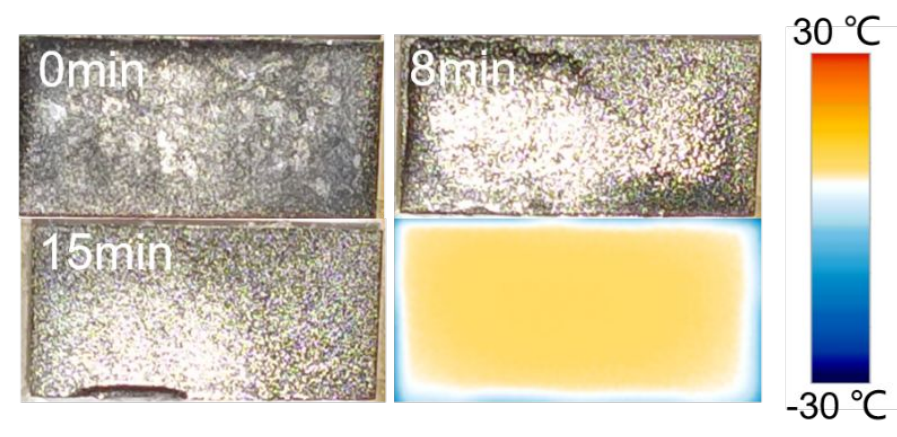

Figure S9 Photographs and infrared image of photothermal deicing process on selfhealed CNTs photothermal coating. 